\title{
Cancer Pain Treated With Rectally Administered Prolonged-Release Oxycodone Hydrochloride Tablets
}

\author{
Hanbing $\mathrm{Wu}^{2}$, Ming Chen ${ }^{3}$, Chunhong $\mathrm{Hu}^{1}$, Xianling Liu ${ }^{1}$, Yawen Gao ${ }^{1}$, \\ Yanliang Sun ${ }^{2}$, Fang $\mathrm{Wu}^{1{ }^{*}}$ \\ ${ }^{1}$ Department of Oncology, the Second Xiangya Hospital of Central South \\ University, Changsha, Hunan 410011, P.R. China; \\ ${ }^{2}$ Department of Oncology, the First People's Hospital of Huaihua City, Huaihua, \\ Hunan 418000, P.R. China; \\ ${ }^{3}$ Department of Gastroenterology, the First People's Hospital of Huaihua City, \\ Huaihua, Hunan 418000, P.R. China.
}

First Author: Hanbing Wu, MM, Chief Physician, Department of Oncology, the First People's Hospital of Huaihua, Huaihua, Hunan 418000, P.R. China;

Joint First Author: Ming Chen, B.S. Chief Physician, Department of Gastroenterology, the First People's Hospital of Huaihua City, Huaihua, Hunan 418000, P.R. China.

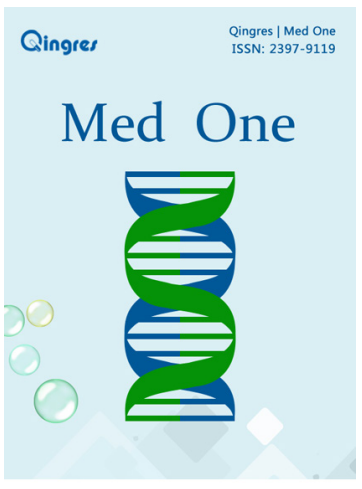

http://mo.qingres.com

\section{GOPEN ACCESS}

DOI: 10.20900/mo.20170008

Received: January 22, 2017

Accepted: March 18, 2017

Published: April 25, 2017

Copyright: ๑2017 Cain et al. This is an open access article distributed under the terms of the Creative Commons Attribution License,which permits unrestricted use, distribution, and reproduction in any medium, provided the original author and source are credited.
Correspondence: Fang Wu, MD, Chief Physician, Department of Oncology, the Second Xiangya Hospital of Central South University, Changsha, Hunan 410011, P.R. China. Email: doctor_wufang@126. com.

\section{ABSTRACT}

Objective: To observe the clinical efficacy and side effects of rectally administered prolonged-release oxycodone hydrochloride tablets on cancer pain.

Methods: Seventy-six patients with moderate-severe cancer pain admitted to April 2010-April 2016 were randomly chosen and equally divided into an experimental group and control group. All patients were each treated with prolonged-release oxycodone hydrochloride tablets. The experimental group patients were administered rectally and the control group were administered orally. Clinical efficacy and side effects were observed for both groups.

Results: There was no statistically significant difference $(p>0.05)$ in the pain scores of the two groups after administration of prolongedrelease oxycodone hydrochloride tablets after $1 \mathrm{~h}, 3 \mathrm{~h}, 1$ day, 3 days 
and 1 week. Gastrointestinal reaction incidence rates for the experimental group was significantly lower than the control group with the difference being statistically significant $(p<0.05)$.

Conclusion: Rectal administration had the same analgesic effects as oral administration, but had fewer side effects and more clinical value.

Keywords: cancer pain; oxycodone hydrochloride prolonged-release tablets; rectal administration.

\section{INTRODUCTION}

Between $35 \%$ and $50 \%$ of patients with clinically malignant tumors will develop pain at various levels. The incidence rate of cancer pain, particularly in advanced cancer patients, is more than $60 \%$, and it is severe and persistent, causing great pain and seriously affecting their quality of life ${ }^{[1]}$. It is a standard treatment for moderate cancer pain patients to orally take opioid analgesics. However, anorexia from cachexia syndrome, aphagosis from intestinal obstructions and even severe vomiting after oral administration occurred in some which affects the application of these analgesics. The use of morphine controlled release tablets for rectal delivery has been reported. At present, there is no opioid sustainedrelease suppository. Cancer patients were treated either orally or rectally with prolonged-release oxycodone hydrochloride tablets. Clinical effects were observed and compared to provide a basis of selecting the manner of clinical administration.

\section{MATERIALS AND METHODS}

\subsection{Clinical data}

Seventy-six patients with moderate-to-severe cancer pain admitted between April 2010 and April 2016 were randomly chosen and equally divided between an experimental group and a control group. In the experimental group there were 21 males and 17 females, aged 37-81 years, with a mean age of $63.8 \pm 7.9$. Twenty-six had with moderate cancer pain and 12 had severe cancer pain. Among them, 10 cases of lung cancer, 6 cases of breast cancer, 5 cases of nasopharyngeal carcinoma, 4 cases of colorectal cancer, 3 cases of liver cancer, 2 cases of esophageal cancer, 2 cases of gastric cancer, 2 cases of cervical cancer, 2 cases of pancreatic cancer, 1 case of tongue cancer, 1 case of renal cell carcinoma. The control group consisted of 23 males and 15 females, aged 38-79, with a mean age of $60.1 \pm 6.9$. Twenty-seven had moderate cancer pain and 11 had severe cancer pain.Among them, 12 cases of lung cancer, 5 cases of colorectal cancer, 4 cases of breast cancer, 4 cases of cervical carcinoma, 2 cases of hepatocellular carcinoma, 2 cases of pancreatic carcinoma, 2 cases of nasopharyngeal carcinoma, 2 cases of gastric cancer, 2 cases of prostate cancer, 1 cases of esophageal carcinoma, 1 case of testicular tumors, 1 case of bladder cancer. A statistical analysis showed that any differences of basic information and pain severity between the two groups was not statistically significant $(p>0.05)$, but was comparable. All were diagnosed as advanced malignant tumors via a clinical examination, imaging, and pathology. The expected survival period was not more than a month. They suffered either moderate or severe pain and had a NRSscore of 5 , or more, points. Some were resistant to the first-stage and second-stage drugs ${ }^{[2]}$ guidelines recommend that moderate-tosevere cancer pain patients take opioids directly for analgesic treatment ${ }^{[3]}$. Mostpatients enrolled after 2014 were treated with prolonged-release oxycodone hydrochloride tablets by titration. Patients with acute abdomen, paralytic ileus or other gastrointestinal diseases were excluded as were those who refused to cooperate with treatment or the clinical survey. All patients in this study were informed of the experimental procedures and voluntarily participated in this experiment and provided written informed consent form. The information was approved by the ethics committee of the Hospital.

\subsection{Methods}

Patients in both groups were each treated with prolonged-release oxycodone hydrochloride tablets. Patients in the experimental group were administered rectally. The process began with a patient emptying a stool before administration, and then was placed in a lateral position on their back with buttocks slightly raised. Slowly and gently using a gloved, and Vaselined, index finger medical personnel tapped the anus to relax it, or injected warm water to wet it, and then inserted the tablets to at least the dentate line. The patient maintained the initial position for 30 min after insertion. Initial dosages were 10-20 $\mathrm{mg} / \mathrm{time}$. Administration was made once every 12 hours. Dosage was gradually increased according to patient-reported pain levels until the pain was relieved or disappeared (NRS score $\leq 3$ points). Any breakthrough pain was treated with a subcutaneous morphine injection. Patients in the experimental group were each treated with $10 \mathrm{ml}$ lactulose thrice daily or wit $0.2 \mathrm{~g}$ phenol phthalide tablets twice daily to prevent constipation. Control group patients were 
orally administered using the same treatment regime as the experimental group.

\subsection{Observation Indicators}

Results of the treatment were evaluated and recorded by both medical personnel and patients as follows: 1) post-treatment pain relief for the two groups used the NRS scoring method ${ }^{[4]}$ : 0 points meant for no pain, 1-3 mild pain, 4-6 moderate pain, and $7-10$ severe pain. Post-administration pain relief rate was evaluated at $1 \mathrm{~h}, 3 \mathrm{~h}, 1$ day, 3 days, and one week. NRS 0 point meant a significant relief, 1-3 points a basic relief, and 4 more unsatisfactory relief. Pain control efficiency was calculated as: significantly relieved cases + basically relieved cases)/enrolled cases x $100 \%$. 2) Side effects were measured a week after administration as: a. nausea and vomiting: refer to the Cancer Hospital of Chinese Academy of Medical Science's gastrointestinal reaction grading criteria of $\mathrm{s}$ : 0 degree meant no nausea and no vomiting, I nausea without vomiting, II nausea with mild vomiting, III vomiting requiring treatment, and IV vomiting beyond the control ${ }^{[5]}$; b. constipation; c. drowsiness; d. psychiatric symptoms such as comfort, trance, etc.); e. urinary retention, muscle tremor, convulsion, respiratory depression, addiction, and other measures. A data statistical analysis and comparison was performed comparing the two groups.

\subsection{Data Processing}

Patient data for both groups was subjected to a statistical analysis using SPSS17.0. Measurement data was expressed as $\bar{x} \pm \mathrm{s}$, $\mathrm{t}$ was used for test; counting data was expressed as $\mathrm{n}(\%), x^{2}$ as used in the test. Confidence interval was $95 \%$ and the test level was 0.05. $p<0.5$ indicating that sample data were significantly different and statistically significant.

\section{RESULTS}

\subsection{Pain Relief Degree Evaluation}

Patient pain scores for the two groups were not significantly different after administration at $1 \mathrm{~h}, 3 \mathrm{~h}$, 1 day, 3 days or a week, $p<0.5$, was not statistically significant (Tables 1. and 2).

Table 1. NRS Score Comparison, $\bar{x} \pm \mathrm{s}$

\begin{tabular}{|c|c|c|c|c|c|c|}
\hline \multirow{2}{*}{ Group } & \multirow[b]{2}{*}{ Casen } & \multicolumn{5}{|c|}{ Elapsed Time } \\
\hline & & $1 \mathrm{~h}$ & $3 h$ & 1 day & 3 days & 1 week \\
\hline Experimental & 38 & $4.24 \pm 1.27$ & $3.73 \pm 1.18$ & $3.06 \pm 1.11$ & $2.02 \pm 1.08$ & $1.62 \pm 0.90$ \\
\hline Control & 38 & $4.34 \pm 1.23$ & $3.82 \pm 1.30$ & $3.14 \pm 0.88$ & $2.14 \pm 0.81$ & $1.91 \pm 0.82$ \\
\hline
\end{tabular}

Note: $p>0.05$ indicates that the sample data were not statistically significant.

Table 2. Comparison of Pain Control Efficiency, n (\%)

\begin{tabular}{|c|c|c|c|c|c|c|c|c|c|c|}
\hline \multirow{2}{*}{$\begin{array}{l}\text { Different } \\
\text { Time } \\
\text { Points }\end{array}$} & \multicolumn{5}{|c|}{ Experimental Group } & \multicolumn{5}{|c|}{ Control Group } \\
\hline & $\begin{array}{c}\text { Case } \\
\text { n }\end{array}$ & $\begin{array}{c}\text { Complete } \\
\text { relief }\end{array}$ & $\begin{array}{l}\text { Basic } \\
\text { relief }\end{array}$ & $\begin{array}{l}\text { Unsatisfactory } \\
\text { relief }\end{array}$ & $\begin{array}{c}\text { Pain relief } \\
\text { efficiency, } \\
\%\end{array}$ & $\begin{array}{c}\text { Cace } \\
\mathbf{n}\end{array}$ & $\begin{array}{l}\text { Complete } \\
\text { relief }\end{array}$ & $\begin{array}{l}\text { Basic } \\
\text { relief }\end{array}$ & $\begin{array}{l}\text { Unsatisfactory } \\
\text { relief }\end{array}$ & $\begin{array}{c}\text { Pain relief } \\
\text { efficiency, } \\
\%\end{array}$ \\
\hline $1 \mathrm{~h}$ & 38 & 6 & 11 & 21 & 44.73 & 38 & 5 & 11 & 22 & 42.10 \\
\hline $3 h$ & 38 & 7 & 13 & 18 & 52.63 & 38 & 7 & 12 & 19 & 50.00 \\
\hline 1 day & 38 & 6 & 18 & 12 & 63.15 & 38 & 5 & 16 & 17 & 55.26 \\
\hline 3 days & 38 & 11 & 22 & 5 & 86.84 & 38 & 9 & 24 & 5 & 86.84 \\
\hline 1 week & 38 & 11 & 20 & 7 & 81.57 & 38 & 12 & 22 & 4 & 89.47 \\
\hline
\end{tabular}

Note: $p>0.05$ indicates that the sample data were not statistically significant. 


\subsection{Observation of adverse drug reactions}

There were 10 cases of nausea and vomiting in the experimental group, including 6 degree-l cases, 3 degree-II cases, and 1 degree-III 1. The control group had 18 instances of nausea and vomiting, including 4 degree-I cases, 5 degree-Il cases, and 9 degree-III cases. The experimental group had
12 instances of constipation and 20 the control group 20. The experimental group had 5 cases of dizziness and drowsiness and the control group 9. The experimental group had 2 cases of psychiatric symptoms and the control group 3 . There were no urinary retention, muscle tremor, convulsion, respiratory depression, addiction or other side effects in either group.

Table 3. Comparison of Administration Side Effects, $\mathrm{n}(\%)$

\begin{tabular}{lccccc}
\hline Group & Case & $\begin{array}{c}\text { Nausea \& } \\
\text { vomiting * }\end{array}$ & Constipation* & Drowsiness* & $\begin{array}{c}\text { Psychiatric } \\
\text { symptom }\end{array}$ \\
\hline Experimental & 38 & $10(26.31)$ & $12(31.57)$ & $5(10.00)$ & $2(3.33)$ \\
Control & 38 & $18(47.36)$ & $20(52.63)$ & $9(20.00)$ & $3(6.67)$ \\
\hline
\end{tabular}

Note: ${ }^{*} p<0.05$ indicates that the sample data is significantly different and statistically significant.

\section{DISCUSSION}

In 1995, American Pain Society (APS) Chairman James N. Campbell proposed pain as the fifth largest vital sign. The International Association for the Study of Pain (IASP) has slightly modified the definition of the cancer pain this year, to be that cancer pain is an uncomfortable, sensitive and emotional experience associated with the actual or potential tissue damage ${ }^{[6]}$. Cancer pain is a major symptom of cancer patients, especially in middle, and mid-advanced, cancer patients. If no timely cancer intervention is performed, it seriously affects patient survival quality. prolonged-release oxycodone hydrochloride tablets are a novel opioid receptor agonist with the more comprehensive effect of exciting the opioid receptor subtypes. It not only affects the formation of $\mu$ receptor, but also acts on $\mathrm{k}$ receptor, having a more ideal analgesic effect on pain including, among others, visceral pain and physical pain ${ }^{[7]}$. Current controlled-release technology was used: it contains $38 \%$ immediate release ingredients that provide an analgesic effect within an hour post-administration, and $62 \%$ sustained-release ingredients that can realize 12 hours of cancer pain control ${ }^{[8]}$. Currently most patient cancer pain can be effectively controlled through the oral administration. However, oral administration is slow and irregular, and efficacy is easily affected by the gastrointestinal functions and gastrointestinal contents. Certain types of patients are unable to receive the drug orally such as those who are unconscious or comatose patients; have pyloric obstruction, or intestinal, obstruction; have severe gastrointestinal reactions resulting from radiotherapy and chemotherapy; have severe vomiting after oral administration. Patients with head and neck cancer will experience oropharyngeal pain during radiotherapy, which may result in the refusal of oral analgesics. For cancer pain patients unable to be orally administered a convenient and effective method for pain relief is important.

This study suggests there are no significant differences $(p>0.05)$ in the pain scores for the patients in the two groups after taking prolongedrelease oxycodone hydrochloride tablets at $1 \mathrm{~h}$, $3 \mathrm{~h}, 1$ day, 3 days, or 1 week, and the results were not statistically significant. Compared to oral administration, rectal administration had the same analgesic effect. Side effect incidence rate in the experimental group was significantly lower than for the control group with the difference being statistically significant $(p<0.05)$. In rectal administration, the medication directly enters the liver via the superior rectal veins. They then enter the systemic circulation after the metabolism by the liver, or the internal iliac vein through the inferior rectal veins and anal veins, and finally enter they enter the systemic circulation directly without passing through the liver via the 
inferior vena cava ${ }^{[9,10]}$. In clinical applications, more than $50 \%$ of the medications do not have to enter the liver, which significantly reduces the first pass effect of the liver, effectively reduces and avoids the impact of the medications on the gastrointestinal tract, and significantly reduces various adverse reactions, especially the gastrointestinal reactions, such as nausea and vomiting. To a certain extent, it reduces the drug for treating vomiting, Economic costs declined slightly. Rectal administration has some shortcomings: rectal administration requires privacy, equipment and supplies such as gloves and Vaseline for lubrication increase treatment costs; patients must maintain a somewhat awkward posture for at least $30 \mathrm{~min}$ after the administration, so it is time-consuming; the medication may be rejected after administration influencing treatment effect and requiring re-administration and cannot be used on patients with diarrhea, severe hemorrhoids or anal fissures.

The results show that oral and rectal administration has the same analgesic effect, but fewer side effects and thus more clinical value making it is especially suitable for the patients with cachexia, or who fail to or are unwilling to accept, oral administration.

\section{REFERENCES}

1. Che L, Pei LJ, Huang YG. Advances in Clinical Application of Opioids. Guangdong Med J. 2015; 21: 3264-3266.

2. Sun Y, Gu WP. Guidelines for Three-Step Pain Relief in Cancer Pains. 2nd Edition. Beijing:
Press of Beijing Medical University; 2002.

3. Luo J, Zhan LL. Introduction to Development of Cancer Pain in China - A Preliminary Study on the New Progress of the Second Edition of Guidelines for NCCN Adult Cancer Pain in 2015. J Chin Phys. 2016; 18: 104-105.

4. Robert BR, Joseph VP, Ronald JT. The Determination and Application of Fixed-Dose Analgesic Combinations for Treating Multimodal Pain. J Pain. 2010;11(8): 701-709.

5. Zhou JC.Practice of Medical Oncology. Beijing: People's Medical Publishing House; 2003.

6. Liu YJ, Cao YD, Liu X. Advances in Study of Opioids in Treatment of Chronic ModerateSevere Cancer Pain. Contemp Med Symp. 2016; 18:104-105.

7. LI SH, Li YH, Ye XW, Lu DY, Chen Y. Oxycodone Hydrochloride Improves the Anxiety and Depression in Patients with Moderate and Severe Cancer Pain. Chin J Pain Med. 2013; 19(7): 387-391.

8. Greiner W, Lehmann K, Earnshaw S, Bug C, Sabatowski R. Economicevaluation of durogesic in moderate to severe, nonmalignant, chronic pain in Germany. Eur J Econ. 2006; 7(4): 290.

9. Xing $X Y$, Chen $Y$. Effects of rectal controlled release morphine sulfate on cancer pain. Pain Clin J. 2008; 4(6): 422-424.

10. Li CG, Huang XE. Clinical Observations on Safety and Efficacy of OxyContin Administered by Rectal Route in Treating Cancer Related Pain. Asian Pac J Cancer Prev. 2011; 12(10): 2477-2478. 